\title{
Minimal Residual Disease Assessment in CLL/SLL: Is It Ready for Routine Clinical Practice?
}

\author{
Presented by Jennifer A. Woyach, MD
}

\begin{abstract}
Minimal residual disease (MRD) in chronic lymphocytic leukemia (CLL) is defined as $<1$ CLL cell per 10,000 leukocytes $\left(0.01 \%\right.$; $\left.<10^{-4}\right)$. Flow cytometry and next-generation sequencing have demonstrated high sensitivity in MRD detection. MRD assessment may help to determine prognosis after fixed-duration regimens; this has been established in the contexts of chemoimmunotherapy and venetoclax/antibody combinations. In the short term, MRD status does not seem to inform prognosis in patients treated with a BTK inhibitor plus venetoclax-based regimens; however, long-term data will be needed to determine whether it is beneficial in this population. Numerous trials have demonstrated that MRD may be used to guide therapy. It is unclear whether using an MRD-guided treatment strategy is better than using fixed-duration therapy; ongoing and future studies are warranted.
\end{abstract}

J Natl Compr Canc Netw 2021;19(11.5):1343-1345 doi: 10.6004/jnccn.2021.5107

\begin{abstract}
"Minimal residual disease [MRD] is a powerful prognostic tool in chronic lymphocytic leukemia [CLL] for chemoimmunotherapy and venetoclax/antibody combinations, and it will likely be applicable to venetoclax/BTK inhibitor therapies," commented Jennifer A. Woyach, MD, Professor of Medicine, The Ohio State University Comprehensive Cancer Center - James Cancer Hospital and Solove Research Institute. Specifically, undetectable MRD (uMRD) has been found to be associated with a longer duration of progression-free survival (PFS). Dr. Woyach presented data evaluating MRD assessment in CLL and small lymphocytic lymphoma (SLL), as well as the methods and appropriate scenarios for the use of MRD testing at the NCCN 2021 Virtual Congress: Hematologic Malignancies.
\end{abstract}

\section{Detection of MRD \\ "[Flow cytometry], for many years, has been the standard by which we assessed for MRD both in clinical practice and in clinical trials," Dr. Woyach commented. "The European Research Initiative on CLL [ERIC] has really taken a lead in harmonizing flow cytometry methods." Their guidelines suggest that MRD may be reliably detected at levels of at least $10^{-4}$ and, in some cases, to $10^{-5}{ }^{1,2}$ Flow cytometry has wide utilization, is relatively inexpensive, and does not require a pretreatment sam- ple; however, it does require a flow cytometry laboratory. Currently, MRD detection by flow cytometry is not approved by the FDA in this clinical setting.}

Real-time quantitative PCR with allele-specific oligonucleotide primers of immunoglobin heavy chain genes (ASO-PCR) is a highly sensitive measure, and it may reliably detect down to levels of $10^{-5} .3$ According to Dr. Woyach, this method is time-consuming and expensive. "This really isn't used clinically in CLL right now," she remarked. "You may see it in the context of clinical studies."

"The last method is next-generation sequencing (NGS). It is a branding called "ClonoSEQ" that is FDAapproved for the detection of MRD in CLL," Dr. Woyach explained. It is very sensitive-it can detect MRD reliably down to levels of $10^{-6}$. Local facilities are not required for testing because the samples have to be sent out centrally. However, a pretreatment sample is required for testing; it is also relatively expensive.

"I think [when to use MRD detection] is still a point that is debatable," Dr. Woyach commented. When using a continuous therapy, such as BTK inhibition, MRD detection has not been found to be beneficial. MRD assessment may help determine prognosis after some fixed-duration regimens, which has been established in the contexts of chemoimmunotherapy and venetoclax/ antibody combinations. ${ }^{4-8}$

\section{Utility of MRD Detection in CLL}

\section{Chemoimmunotherapy and Venetoclax/Antibody} Combinations

"The first demonstration of the utility of MRD testing in CLL came from studies of fludarabine, cyclophosphamide, 
and rituximab," Dr. Woyach remarked. In 2 clinical trials, ${ }^{4,5}$ patients with uMRD who were administered fludarabine + cyclophosphamide experienced longer durations of PFS; this appeared to hold true regardless of whether rituximab was added to the regimen. "It was first demonstrated that approximately $60 \%$ [of patients with the $I G H V$-mutated phenotype treated with fludarabine + cyclophosphamide/rituximab] would be cured by the regimen," Dr. Woyach commented. "We can see that when you look at patients by MRD status..., those who are $I G H V$-mutated and MRD-negative at the end of treatment have an approximately $80 \%$ chance of being cured with this one therapy. That is really phenomenal for a disease that was widely considered incurable."

The phase III MURANO study led to the FDA approval of venetoclax + rituximab for the treatment of relapsed CLL. ${ }^{6}$ In terms of PFS, this regimen was found to be superior to bendamustine + rituximab. According to Dr. Woyach, in many patients uMRD was achieved relatively early. The 18-month PFS rates in patients with undetectable, low, and high levels of MRD were $90 \%$, $64 \%$, and $8 \%$, respectively. ${ }^{7}$

In the phase III CLL14 trial, patients with uMRD experienced a longer duration of PFS at the end of treatment than those with high or low levels of MRD; this held true regardless of whether they were administered initial therapy with obinutuzumab + venetoclax or chlorambucil. ${ }^{8}$ Dr. Woyach explained that there was a high rate of UMRD with obinutuzumab + venetoclax. Based on these data, the FDA approved the use of venetoclax + obinutuzumab given for 1 year in this clinical context.

"It is clear that uMRD predicts for PFS in patients treated with chemoimmunotherapy, as well as those treated with venetoclax + anti-CD20 antibodies," Dr. Woyach remarked. "Serial measurements of MRD following therapy reliably predict [disease] progression events, although with a delay." She explained that the level of sensitivity needed for the detection of MRD is still being considered.

\section{Venetoclax + BTK inhibitor Therapy}

In a clinical trial of ibrutinib + venetoclax and obinutuzumab, the median PFS rate was approximately $95 \%$ after 3 years. ${ }^{9}$ Approximately $66 \%$ of patients achieved uMRD at the end of treatment. Updated data from the phase II trial of frontline ibrutinib + venetoclax demonstrated that the rate of uMRD was $56 \%$ with 12 cycles of therapy and $66 \%$ with 24 cycles, with a best response of $75 \%$. The 3-year PFS rate was $93 \% .^{10}$

Data from the fixed-duration cohort of the phase II CAPTIVATE trial revealed a $24-$ month PFS rate of $95 \%$ in patients treated with ibrutinib + venetoclax. ${ }^{11}$ The multicenter phase III GLOW study also evaluated this regimen and found that ibrutinib + venetoclax was superior to chlorambucil + obinutuzumab in terms of PFS. The rates of UMRD were $55 \%$ and $52 \%$ in the peripheral blood and bone marrow, respectively. According to Dr. Woyach, data establishing the relationship between PFS and MRD status at the end of therapy have not yet been released. ${ }^{12}$

"In the short term, MRD status does not seem to help inform the prognosis for BTK inhibitor + venetoclaxbased regimens, because the patients do so well," Dr. Woyach commented. "Long-term data will be needed to determine if this is helpful."

\section{Can MRD Guide Therapeutic Strategy?}

In the phase II trial of frontline ibrutinib + venetoclax, patients were permitted to continue therapy if they remained MRD-positive after 24 cycles. $^{10}$ "We have seen progressive increases in the uMRD rate," Dr. Woyach remarked. "[This suggests] that maybe this is a helpful strategy.”

In the phase II CAPTIVATE trial, after approximately 1 year of treatment with ibrutinib + venetoclax, the MRD cohort was randomized to a maintenance therapy regimen based on MRD status. ${ }^{13}$ Patients with confirmed uMRD (MRD-negative cohort) were randomized to maintenance therapy with ibrutinib or placebo, whereas those without a confirmed uMRD (MRD-positive cohort) were assigned to maintenance therapy with either ibrutinib or ibrutinib + venetoclax. In the MRD-negative cohort, the 30 -month PFS rates were $100 \%$ and $95 \%$ for ibrutinib and placebo, respectively. In the MRD-positive cohort, the 30-month PFS rates were $96.7 \%$ and $95.2 \%$ for patients assigned to maintenance therapy with ibrutinib and ibrutinib + venetoclax, respectively. ${ }^{14}$

"Longer-term data are going to be needed to help us decide whether [patients with uMRD] benefit from additional treatment, and whether there is any advantage to continuation of doublet therapy for those who are MRDpositive," Dr. Woyach remarked.

The phase II CLARITY trial also used an MRDguided treatment strategy. ${ }^{15}$ After 14 months of treatment with ibrutinib + venetoclax, patients with MRDpositive relapsed or refractory CLL continued therapy; those with UMRD continued treatment with ibrutinib alone. The rates of UMRD were $40 \%$ and $48 \%$ at 14 and 26 months, respectively; according to Dr. Woyach, this suggests there may be an advantage to the continuation of therapy. The 3 -year PFS rate was $96 \%{ }^{16}$

An MRD-guided discontinuation strategy was used in the phase II BOVen trial of treatment-nave patients with CLL who received the second-generation BTK inhibitor zanubrutinib + obinutuzumab and venetoclax; when patients achieved uMRD in the bone marrow, they discontinued therapy at a prespecified time. A total of $77 \%$ of patients discontinued therapy at a median of 10 months. ${ }^{17}$ No disease progression or recurrent MRD was observed in 
this study population; however, Dr. Woyach noted there was a short duration of follow-up.

"Very clearly, [we can use MRD to guide therapy]. It has been done in a number of clinical trials," she commented. "What remains unclear at this point is whether that is a better strategy than using fixed-duration [therapy] and, unfortunately, we do not have that answer just yet."

\section{MRD Testing Recommendations}

"I generally would order [MRD testing] following a venetoclax-based therapy, specifically venetoclax plus an antiCD20 antibody," Dr. Woyach remarked. "However, I do not change my management based on the results."

In patients who received a venetoclax-based regimen following disease progression with a BTK inhibitor, Dr. Woyach recommended testing for MRD after 2 years. Continuation of treatment should be considered based on the results, she said.
"There have been a number of clinical trials that have done MRD testing in both the blood and the bone marrow; depending on the studies they have reported concordance rates of 75 to $90 \%$," she commented. "However, most of the studies that have modified therapy as a result of MRD have used bone marrow." Dr. Woyach recommended testing for MRD in the peripheral blood first and, if this is negative, MRD testing in the bone marrow can be considered.

Disclosures: Dr. Woyach has disclosed receiving consulting fees from AbbVie, Inc., AstraZeneca Pharmaceuticals LP, BeiGene, Eli Lilly and Company, Janssen Pharmaceutica Products, LP, and Pharmacyclics; receiving grant/research support from AbbVie, Inc., Janssen Pharmaceutica Products, LP, Karyopharm Therapeutics, MorphoSys AG, Pharmacyclics, and Schrodinger; and serving as a scientific advisor for Newave.

Correspondence: Jennifer A. Woyach, MD, The Ohio State University Comprehensive Cancer Center - James Cancer Hospital and Solove Research Institute, 410 West 10th Avenue, Columbus, OH 43210. Email: Jennifer.Woyach@osumc.edu

\section{References}

1. Rawstron AC, Fazi C, Agathangelidis A, et al. A complementary role of multiparameter flow cytometry and high-throughput sequencing for minimal residual disease detection in chronic lymphocytic leukemia: an European Research Initiative on CLL study. Leukemia 2016;30:929-936.

2. Rawstron AC, Kreuzer KA, Soosapilla A, et al. Reproducible diagnosis of chronic lymphocytic leukemia by flow cytometry: an European Research Initiative on CLL (ERIC) \& European Society for Clinical Cell Analysis (ESCCA) harmonisation project. Cytometry B Clin Cytom 2018;94B:121-128.

3. Raponi S, Starza ID, De Propris MS, et al. Minimal residual disease monitoring in chronic lymphocytic leukaemia patients: a comparative analysis of flow cytometry and ASO IgH RQ-PCR. Br J Haematol 2014;166:360-368.

4. Thompson PA. MRD negativity as a surrogate for PFS in CLL? Blood 2018;131:943-944.

5. Dimier N, Delmar P, Ward C, et al. A model for predicting effect of treatment on progression-free survival using MRD as a surrogate end point in CLL. Blood 2018;131:955-962.

6. Kater AP, Seymour JF, Hillmen P, et al. Fixed duration of venetoclax-rituxi$\mathrm{mab}$ in relapsed/refractory chronic lymphocytic leukemia eradicates minimal residual disease and prolongs survival: post-treatment follow-up of the MURANO phase III study. J Clin Oncol 2019;37:269-277.

7. Kater AP, Wu JQ, Kipps T, et al. Venetoclax plus rituximab in relapsed chronic lymphocytic leukemia: 4-year results and evaluation of impact of genomic complexity and gene mutations from the MURANO phase III study. J Clin Oncol 2020;38:4042-4054.

8. Al-Sawaf $\mathrm{O}$, Zhang $\mathrm{C}$, Tandon $\mathrm{M}$, et al. Venetoclax plus obinutuzumab versus chlorambucil plus obinutuzumab for previously untreated chronic lymphocytic leukaemia (CLL14): follow-up results from a multicentre, open-label, randomised, phase 3 trial. Lancet Oncol 2020;21:1188-1200.

9. Rogers KA, Huang Y, Ruppert AS, et al. Phase II study of combination obinutuzumab, ibrutinib, and venetoclax in treatment-naive and relapsed or refractory chronic lymphocytic leukemia. J Clin Oncol 2020;38:3626-3637.
10. Jain $\mathrm{N}$, Keating $\mathrm{M}$, Thompson $\mathrm{P}$, et al. Ibrutinib plus venetoclax for firstline treatment of chronic lymphocytic leukemia a nonrandomized phase 2 trial. JAMA Oncol 2021;7:1213-1219.

11. Allan JN, Wierda WG, Siddiqi T, et al. Primary analysis of the fixed-duration cohort from the phase 2 CAPTIVATE study of first-line ibrutinib + venetoclax for chronic lymphocytic leukemia/small lymphocytic lymphoma [abstract]. Presented at the European Hematology Association (EHA) Congress 2021; June 9-17, 2021. Abstract S147.

12. Kater A, Owen C, Moreno C, et al. Fixed-duration ibrutinib and venetoclax versus chlorambucil plus obinutuzumab for first-line chronic lymphocytic leukemia: primary analysis of the phase $3 \mathrm{GLOW}$ study [abstract]. Presented at the European Hematology Association (EHA) Congress 2021; June 9-17, 2021. Abstract LB1902.

13. Wierda WG, Tam CS, Allan JN, et al. Ibrutinib plus venetoclax for firstline treatment of chronic lymphocytic leukemia/small lymphocytic lymphoma: 1-year disease-free survival results from the MRD cohort of the phase 2 CAPTIVATE study [abstract]. Blood 2020;136:16-17.

14. Siddiqi T, Tam CS, Allan JN, et al. First-line ibrutinib + venetoclax for patients with chronic lymphocytic leukemia/small lymphocytic lymphoma: efficacy and safety results from captivate MRD cohort [abstract]. Presented at the European Hematology Association (EHA) Congress 2020; June 11-21, 2020. Abstract S158.

15. Hillmen $\mathrm{P}$, Rawstron AC, Brock K, et al. Ibrutinib plus venetoclax in relapsed/refractory chronic lymphocytic leukemia: the CLARITY study. J Clin Oncol 2019;37:2722-2729.

16. Hillmen $\mathrm{P}$, Boucher $\mathrm{RH}$, Webster $\mathrm{N}$, et al. Continued long term responses to ibrutinib + venetoclax treatment for relapsed/refractory CLL in the blood cancer UK TAP Clarity Trial [abstract]. Blood 2020;136:17-18.

17. Soumerai JD, Mato AR, Carter J, et al. MRD-driven time limited therapy with zanubrutinib, obinutuzumab, and venetoclax (BOVen) in previously untreated chronic lymphocytic leukemia [abstract]. Presented at the 62nd American Society of Hematology (ASH) Annual Meeting and Exposition; December 5-8, 2020. Abstract 1307. 